\title{
LUBRICATION THEORY FOR $n$-LAYER THIN-FILM FLOW WITH APPLICATIONS TO MULTILAYER EXTRUSION AND COATING
}

\author{
N. R. ANTURKAR, T. C. PAPANASTASIOU ${ }^{\dagger}$ and J. O. WILKES \\ Department of Chemical Engineering, University of Michigan, Ann Arbor, MT 48109, U.S.A.
}

(First received 12 June 1989; accepted in revised form 4 December 1989)

\begin{abstract}
Two-dimensional, $n$-layer isothermal flows of Newtonian liquids are analyzed by means of the Reynolds lubrication approximation (Phil. Trans. $R$. Soc. 177, 157, 1886). For algebraic simplicity, the coordinates are transformed for individual layers. The resulting governing equations are solved for multilayer coating and for multilayer extrusion by the Galerkin/finite-element method and Newton iteration together with an implicit finite-difference scheme for the time-dependent analysis. The number of unknowns in the finite-element analysis is reduced by appropriately decoupling some of the equations. In the steady-state analysis, the thickness profile of each layer is estimated for given flow rates of individual layers over a range of capillary numbers between $10^{-6}$ and 1000 and a range of viscosity ratios between 0.001 and 20 . In coating, the surface tension of the outer layer was found to have a more pronounced effect on the steady-state solution than the surface tensions of the inner layers. For initial sinusoidal disturbances of various wavelengths and amplitudes, the flow of up to seven layers with various configurations was found to be stable for negligible inertia effects.
\end{abstract}

\section{INTRODUCTION}

There are various ways of modifying physical properties of polymers. Frequently, however, single polymers cannot satisfy all end-uses economically. Therefore, multilayer plastic films are used. Each layer of these composite materials provides a specific end-use characteristic such as oxygen and moisture barrier, thermoformability, mechanical attributes, and heat sealability to the product. Multilayer polymer films and sheets with combinations of these end-uses find applications in food packaging, in lamination to paper and other decorative uses (Schrenk and Alfrey, 1978), and in multilayer coating (Kistler and Scriven, 1984).

Multilayer polymer films and sheets are manufactured by a tubular blown-film process (Schrenk and Alfrey, 1973) or by a flat-die chill-roll process (Schrenk, 1974). In the case of extrusion through a flat die, the film is drawn down through a narrow slit at the outlet of the die and is subsequently quenched below its solidification temperature on a chill-roll. The modern and more widely used method has a single-manifold die. At the inlet of the die, a feedblock is attached that introduces a prearranged multilayer melt stream (see Fig. 1). The feedblock method is more popular than other available methods because of its versatility in accommodating a large number of layers.

Similar to multilayer extrusion, coating the surface of a substrate with multiple layers of liquids is important in various scientific and industrial applications. The five commonly used devices that are suitable for premetered, precision coating are: slot coating, knife coating, roll coating, reverse-roll coating, and slide

${ }^{\dagger}$ To whom correspondence should be addressed. coating (Bixler, 1982). All of them can be used for multilayer coating as well. A schematic diagram of one such arrangement of slot coating is shown in Fig. 2.

Thickness profiles and pressure drops across the pipes and channels in two and three layers have been extensively studied, theoretically and experimentally, by several researchers such as $Y u$ and Han (1973), White et al. (1972), Han and Kim (1976) and Southern and Ballman (1973). These studies were either motivated to reduce the pressure drop or to study the interface deformation in the form of encapsulation of the viscous liquid by the liquid of low viscosity. The analyses of Newtonian, power-law, and viscoelastic liquids have indicated that the important parameters which affect the thicknesses and the pressure drops are viscosity ratios and elasticities of the liquids. The effect of the viscosity stratification on the stability of multilayer flows have also been investigated (Yih, 1967; Joseph et al., 1984; Wong and Jeng, 1987; Waters, 1983). There are very few studies on multilayer coating (Kistler and Scriven, 1984). However, coating of a single layer on a liquid is extensively studied for predicting surface profiles and for predicting unstable conditions (Pearson, 1960; Bixler, 1982; Coyle et al., 1986).

A major limitation of existing theoretical and experimental works on multilayer flows is that no more than three symmetric layers have been analyzed theoretically. Since, at present, many products of multilayer films and sheets involve more than three layers, it is physically more relevant to develop a general model for analyzing the flow of $n$ layers. Here, a general model is developed for isothermal $n$-layer flow of Newtonian liquids that accounts for any numbet of layers and surface tension effects. The model is based on the well-known Reynolds lubrication theory 
(a) EXTRUDER B
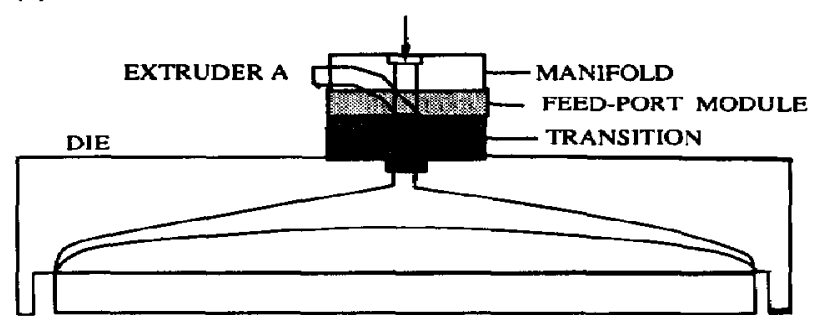

(b)

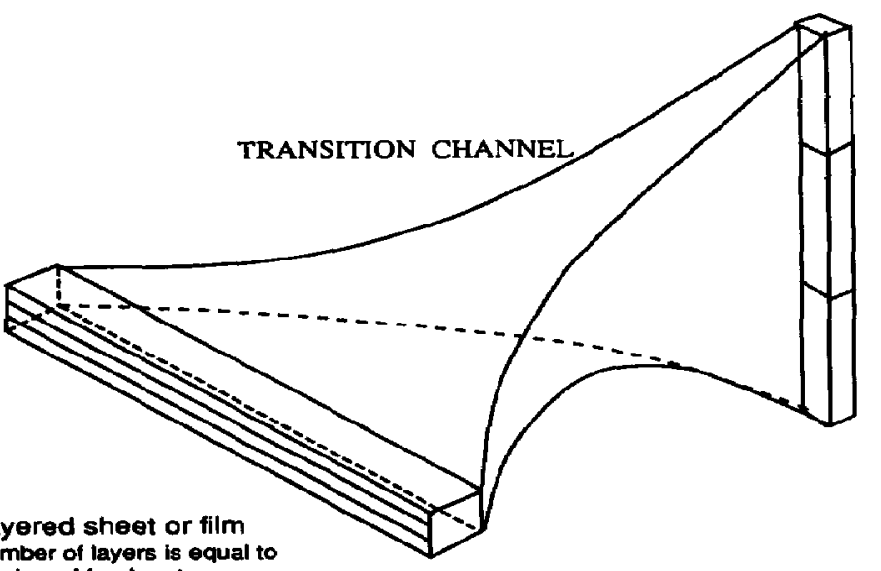

DIRECTION

OF FLOW

Number of leyers is equal to

number of teed ports.

Fig. 1. Multilayer extrusion using single-manifold die (Schrenk and Alfrey, 1978).

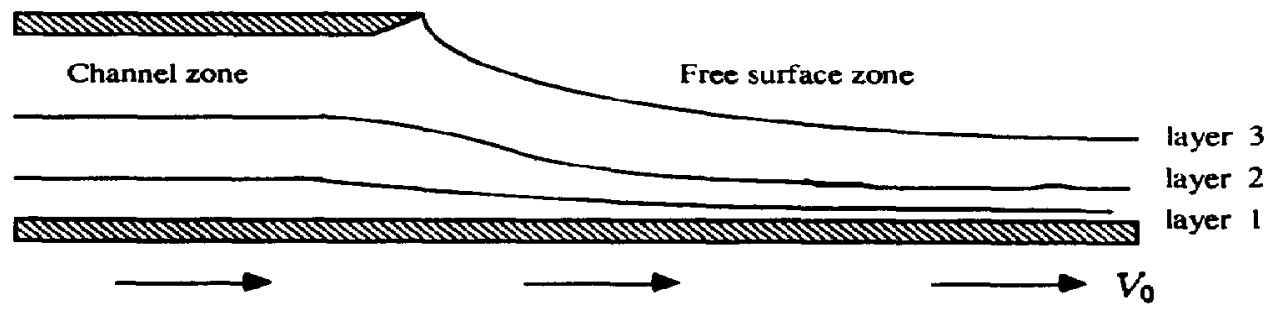

Fig. 2. A schematic diagram of multilayer slot coating.

(1886) and hence it is applicable only to thin-film flows in the absence of recirculation. The same equations can be used for multilayer coating as well as for multilayer extrusion by changing the boundary conditions. The Galerkin/finite-element method is used with Newton iteration to solve the steady-state equations. For the time-dependent analysis with initial sinusoidal disturbances of various wavelengths and amplitudes, an implicit method is used and the stability of the processes is investigated within the limitations of lubrication approximation, the most severe being its validity only for negligible inertia terms. Although the applications of multilayer extrusion and multilayer coating often involve non-Newtonian liquids, the analysis in this paper is developed for Newtonian liquids which is also a first step in analyzing non-Newtonian flows.

Despite its limitations, the lubrication approximation is attractive in the analysis of almost rectilinear flows (confined as well as free surface flows) because it is simple, in many instances manageable by analytical tools, and in numerical modeling, it considerably reduces the number of involved unknowns.

\section{GOVERNING EQUATIONS}

The governing equations are derived with the assumption that the total thickness of all the layers is much smaller than the dimension along the direction of flow, which is reasonable for the industrial applications of multilayer extrusion and of multilayer coating involving thin films. Then, the Reynolds lubrication approximation can be implemented to simplify the equations of change. This approach is fairly standard and is described in various textbooks on liquid mechanics (e.g., Tipei, 1962; Langlois, 1964; Batchelor, 1967). Another assumption made in the analysis is that the process is isothermal. In most situations, the 
temperature variation across the domain of flow is small and the drying or the solidification of the polymer film is carried out away in the downstream direction.

An important step in the development of the theory is to transform the coordinates in such a way that the origin is shifted along the $z$-direction for each layer as shown in Fig. 3. Due to this transformation, the $n$-layer equations become algebraically tractable and Reynolds' equations result naturally from the analysis.

The dimensionless variables after stretching are:

$$
\left.\begin{array}{lll}
\text { coordinates: } & x^{*}=x / L, & z_{i}^{*}=z_{i} /(L \varepsilon), \\
\text { velocities: } & v_{x_{i}}^{*}=v_{x_{i}} / v_{0 *} & v_{x_{1}}^{*}=v_{x_{i}} /\left(v_{0} \varepsilon\right), \\
\text { pressures: } & p_{i}^{*}=p_{i} /\left(\frac{\mu_{i} v_{0}}{\varepsilon^{2} L}\right), & \\
\text { time: } & t^{*}=t /\left(L / v_{0}\right), &
\end{array}\right\}
$$

where the superscript * denotes the dimensionless variables and the subscript $i$ denotes the variables of the ith layer. $x$ and $z_{i}$ are the coordinates, $v_{x_{t}}$ and $v_{z_{t}}$ the velocities in the $x$ and $z_{l}$ directions, $p_{l}$ the pressure and $t$ the time. The variables are normalized by the average velocity $v_{0}$, at the inlet for coextrusion and at the outlet for coating, the viscosity $\mu_{i}$ and the length of the die $L$ in the case of coextrusion or the effective length $L$ for uniform film in the case of coating. The aspect ratio $\varepsilon$ equals $H_{o} / L$, where $H_{0}$ is the total thickness of the film, defined to be at the inlet for coextrusion and at the outlet for coating. The pressure scale is proportional to $\varepsilon^{-2}$ to obtain consistent equations of motion.

The dimensionless variables are substituted in the Navier-Stokes equations of momentum for each layer and an order-of-magnitude analysis is performed. The resulting dimensionless equations, with superscript * hereafter suppressed, are:

$$
\begin{gathered}
\frac{\partial p_{i}}{\partial z_{i}}=0 \\
-\frac{\partial p_{i}}{\partial x}+\frac{\partial^{2} v_{x_{i}}}{\partial z_{i}^{2}}=0, \quad i=1,2, \ldots, n .
\end{gathered}
$$

Equation (2) shows that the pressure $p_{t}$ remains constant across the thickness of the ith layer. By integrating eq. (3) twice across the thickness of the $i$ th layer, the expression for the velocity $v_{x_{i}}$ is

$$
v_{x_{i}}=A_{i}(x, t)+B_{i}(x, t) z_{i}+\frac{z_{i}^{2}}{2} \frac{\partial p_{i}}{\partial x}
$$

where $A_{i}$ and $B_{i}$ are integration constants. Equation (4) shows that the Reynolds lubrication approximation implies a parabolic velocity profile across the thickness.

The boundary conditions at the wall and at the interfaces are as follows.

1. At $z_{1}=0$, a no-slip boundary condition at a solid surface gives

$$
\begin{array}{ll}
v_{x_{1}}=0 & \text { (for coextrusion), or } \\
v_{x_{1}}=1 & \text { (for coating). }
\end{array}
$$

In the case of coating, the dimensional relationship of $Q_{0}=H_{0} V_{0}$ holds, where $V_{0}$ is the dimensional velocity of the substrate. Therefore, the dimensionless substrate velocity equals 1 .

2. At $z_{i}=h_{i}$ and $z_{i+1}=0$,

$v_{x_{i}}=v_{x_{i+1}} \quad$ (continuity of velocity at the interface), $\partial v_{x_{i}} / \partial z_{i}=M_{i} \partial v_{x_{i+1}} / \partial z_{i+1}$

(continuity of shear stress at the interface),

$$
i=1,2, \ldots, n-1,
$$

where $M_{i}$ is the viscosity ratio $\mu_{i+1} / \mu_{i}$.

3 At $z_{n}=h_{n}$.

$v_{x_{x}}=0$ (no-slip boundary condition at the solid surface for coextrusion), or

$\partial v_{x_{n}} / \partial z_{n}=0$ (zero shear stress at the free surface for coating).

The boundary conditions of shear stress at the interfaces and free surface are consistent with the lubrication approximation, which can accommodate small surface gradients. These boundary conditions are derived in Appendix $A$.

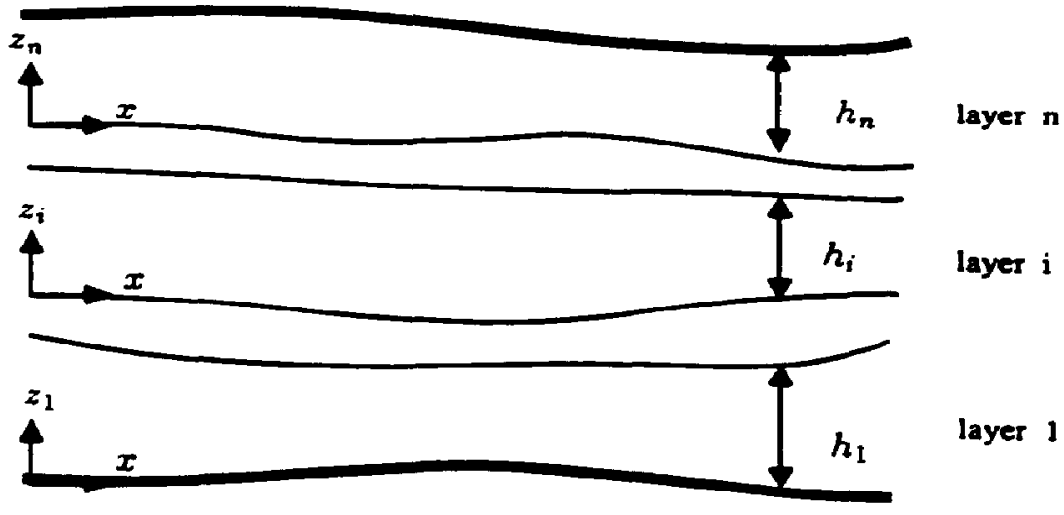

Fig. 3. The local coordinate axes of n-layer flow. 
When these $2 n$ boundary conditions are substituted into eq. (4), we obtain $2 n$ simultaneous equations, in as many unknowns $A_{i}$ and $B_{i}$.

1. For coating:

$\boldsymbol{A}_{1}$

$$
\begin{gathered}
-A_{i}-h_{i} B_{i}+A_{i+1} \\
-B_{i}
\end{gathered}
$$

2. For coextrusion:

$A_{1}$

$$
\begin{gathered}
-A_{i}-h_{i} B_{i}+A_{i+1} \\
-B_{i}
\end{gathered}
$$$$
+
$$$$
M_{i} B_{i+1}
$$$$
\boldsymbol{A}_{n}
$$$$
+M_{i} B_{i+1}
$$

By integrating the continuity equation across the thickness of the ith layer, and then combining it with the equation of the principle of conservation of mass in the $i$ th layer, the resulting equation is:

$$
\frac{\partial}{\partial t}\left(\rho_{i} h_{i}\right)+\int_{0}^{h_{i}} \rho_{i} v_{x_{i}} \mathrm{~d} z_{i}=0, \quad i=1,2, \ldots, n,
$$

where $\rho_{i}$ is the density of the liquid in the ith layer. By substituting eq. (4) in eq. (7), and noting that for incompressible liquids the density $\rho_{i}$ can be eliminated from eq. (7), the Reynolds equation for each layer is

$\frac{\partial h_{i}}{\partial t}+\frac{\partial}{\partial x}\left[A_{i} h_{i}+B_{i} \frac{h_{i}^{2}}{2}+\frac{h_{l}^{3}}{6} \frac{\partial p_{i}}{\partial x}\right]=0$,

$$
i=1,2, \ldots, n \text {. }
$$

For $n$ layers, there are $n$ such coupled equations. However, for each layer $i$, there are two unknowns, the thickness $h_{i}$ and the pressure $p_{i}$. Therefore, $n$ additional equations are required. The first $(n-1)$ equations are obtained by using the normal-stress boundary conditions for the $(n-1)$ interfaces. The remaining equation is derived from the fact that the sum of the thicknesses of all the layers must be equal to the gap between the die walls in the case of coextrusion, and from the normal-stress boundary condition of the free surface in the case of coating.

According to the normal-stress boundary condition, a pressure jump across the interface, in the form of dimensionless variables, is given by:

$$
p_{i}-M_{t} p_{i+1}=\frac{\varepsilon^{2} L}{C a_{i}}\left(2 H^{*}\right), \quad i=1,2, \ldots, n-1,
$$

$$
\begin{array}{cc}
= & 1 \\
= & \left(h_{i}^{2} / 2\right)\left(\partial p_{i} / \partial x\right) \\
= & h_{i}\left(\partial p_{i} / \partial x\right) \\
-B_{n}= & h_{n}\left(\partial p_{n} / \partial x\right) \\
& i=1,2, \ldots, n-1 .
\end{array}
$$

$$
\begin{array}{rc}
= & 0 \\
= & \left(h_{i}^{2} / 2\right)\left(\partial p_{i} / \partial x\right) \\
= & h_{i}\left(\partial p_{i} / \partial x\right) \\
-h_{n} B_{n}= & \left(h_{n}^{2} / 2\right)\left(\partial p_{n} / \partial x\right) \\
& i=1,2, \ldots, n-1
\end{array}
$$

where $C a_{i}$ is the capillary number equal to $v_{0} \mu_{i} / \sigma_{i}, \sigma_{i}$ is the surface tension between layers $i$ and $i+1$, and $2 \mathrm{H}^{*}$ is the curvature in the form of dimensionless variables. The expression for the curvature of the translationally symmetric film is

$$
2 H^{*}=\frac{-\varepsilon \frac{\partial^{2} h_{i}}{\partial x^{2}}}{L\left(1+\left(\varepsilon \frac{\partial h_{i}}{\partial x}\right)^{2}\right)^{3 / 2}} \approx-\frac{\varepsilon}{L} \frac{\partial^{2} h_{i}}{\partial x^{2}},
$$

where the term of $O\left(\varepsilon^{2}\right)$ in the denominator of the right-hand side is neglected. Therefore, from eqs (9) and (10), the normal-stress boundary condition is

$$
p_{i}-M_{i} p_{i+1}=-\frac{\varepsilon^{3}}{C a_{i}} \frac{\partial^{2} h_{i}}{\partial x^{2}}, \quad i=1,2, \ldots, n-1 .
$$

Equation (11) implies that unless $C a_{i} /\left(\partial^{2} h_{i} / \partial x^{2}\right)$ is of $O\left(\varepsilon^{3}\right)$ or smaller, the surface tension effects can be neglected. In coextrusion of polymer melts, the surface tension $\sigma_{i}$ is generally not high enough so that $C a_{i} /\left(\partial^{2} h_{i} / \partial x^{2}\right)$ is of $O\left(\varepsilon^{3}\right)$. However, to generalize the governing equations and to study the high surface tension effects, we keep the term on the right-hand side of eq. (11).

The remaining equation is:

$$
\begin{array}{ll}
\sum_{i=1}^{n} h_{i}= & \begin{array}{l}
\text { (no-slip boundary condition at the } \\
\text { solid surface for coextrusion), or }
\end{array} \\
p_{n}=-\left(\varepsilon^{3} / C a_{n}\right)\left(\partial^{2} h_{n} / \partial x^{2}\right) & \begin{array}{l}
{[\text { eq. (9) at the free surface for }} \\
\text { coating], }
\end{array}
\end{array}
$$


where $f^{*}(x)$ is the ratio of the thickness of the die at any location to the thickness of the die at the inlet.

Thus, eqs (8), (11), and (12) provide $2 n$ equations involving $2 n$ unknowns, $h_{i}$ and $p_{i}$, for $n$-layer flow. The other $2 n$ unknowns $A_{i}$ and $B_{i}$, involved in eq. (8), are obtained from eq. (5) or (6).

In coextrusion, the pressure at the outlet of the die is atmospheric and hence equal to zero. Yu and Han (1973) have reported an experimental observation of positive pressure at the outlet of the rectangular die for polymeric liquids. However, this exit pressure is small compared to the pressure at the inlet of the die. For coating, the boundary condition of zero pressure gradient far downstream of the flow is well established. Therefore,

\section{SOLUTION OF EQUATIONS}

The Galerkin/finite-element method, which is used in this analysis, is well described in several books such as in Strang and Fix (1973). Here, we merely outline the procedure of the finite-element formulation, emphasizing the efforts made in reducing the number of unknowns by decoupling some of the equations.

The unknowns $h_{i}$ and $p_{i}$ are expanded in a suitable set of one-dimensional quadratic finite-element basis functions $\phi_{j}(\xi(x))$ :

$$
\left.\begin{array}{l}
h_{i}(x, t)=\sum_{j=1}^{m n} h_{i j}(t) \phi_{j}(\xi(x)), \\
p_{i}(x, t)=\sum_{j=1}^{m n} p_{i j}(t) \phi_{j}(\xi(x)), \quad i=1,2, \ldots, n,
\end{array}\right\} .
$$

$$
\left.\begin{array}{lll}
\text { at } x=1.0, & p_{i}=0 \\
\text { at } x=x_{\mathrm{f}}, & \partial p_{i} / \partial x=0 & \text { (for coextrusion), or } \\
\text { (for coating), } & i=1,2, \ldots, n,
\end{array}\right\}
$$

where $x_{f}$ is the dimensionless distance along the flow where the films become virtually planar.

In the lubrication approximation, the flow rate or the thickness of each layer can be specified at the inlet of the flow. Under processing conditions, however, the flow rate for cach layer is expected to be known at the inlet instead of its thickness. Therefore, we specify the flow rates of each layer as the inlet boundary conditions,

$$
\text { at } x=0: \quad Q_{i}=q_{i}, \quad i=1,2, \ldots, n,
$$

where $Q_{i}$ is the dimensionless flow rate per unit width and $q_{i}$ are prescribed constants along with the constraint:

$$
\sum_{i=1}^{n} q_{i}=1 \text {, }
$$

due to the conservation of mass. In the case of coextrusion, the flow is assumed to be fully developed at the inlet of the die. Therefore,

$$
\text { at } x=0: \quad \frac{\partial h_{i}}{\partial x}=0 \quad i=1,2, \ldots, n .
$$

For coating, the total thickness at $x=0$ is additionally assumed to be fixed. Then,

$$
\text { at } x=0, \quad \sum_{i=1}^{n} h_{i}=H_{1} \text {. }
$$

Thus, the unknowns in the equations are the thicknesses $h_{i}$, the pressures $p_{i}$, and the constants $A_{i}$ and $B_{i}$. The solution of the system of equations for multilayer extrusion or for multilayer coating depends on the following dimensionless groups and parameters:

\section{Capillary number}

Viscosity ratio

Aspect ratio

Flow rate per unit width at the inlet

Spatial variation of the gap between the die walls (for coextrusion)

Thickness of the slot at $x=0$ (for coating) where $n n$ is the number of nodes and $\xi$ the isoparametric coordinate such that $\mathscr{F}=(\xi: 0$ $\leqslant \xi \leqslant+1$ ). Each of the elements in the flow domain is mapped through the isoparametric relation:

$$
x=\sum_{j=1}^{3} x_{j} \phi_{j}(\xi(x)),
$$

on the interval $\mathscr{F}$, where $x_{j}$ is the coordinate of the $j$ th node. Since for each node there are $2 n$ unknowns, $h_{i}$ and $p_{i}$, the unknowns or degrees of freedom in one element are $6 n$, where $n$ is the number of layers. $A$ procedure to evaluate the additional unknowns $A_{i}$ and $B_{i}$ is outlined subsequently.

In the Galerkin/finite-element method, the basis functions themselves are used as weighting functions in computing the residuals. Then the residual of the Reynolds lubrication equation in the $i$ th layer [see eq. (8)] is

$$
\begin{aligned}
R_{i j}^{(R)}= & \int_{0}^{1}\left[\frac{\partial h_{i}}{\partial t}+\frac{\partial}{\partial x}\left(A_{i} h_{i}+\frac{B_{i} h_{i}^{2}}{2}\right.\right. \\
& \left.\left.+\frac{h_{i}^{3}}{6} \frac{\partial p_{i}}{\partial x}\right)\right] \phi_{j} \frac{\mathrm{d} x}{\mathrm{~d} \xi} \mathrm{d} \xi, \quad i=1,2, \ldots, n .
\end{aligned}
$$

The evolution of flow with time is represented by the first term, discretized by backward-differencing in the above equation. The choice of this implicit method is primarily due to its well-known stability. To lower the order of the differential equation and to impose the natural boundary conditions, the divergence theorem is applied to the second term of the above equation.

$$
\begin{array}{ll}
C a_{i}=\mu_{i} v_{0} / \sigma_{i}, & i=1,2, \ldots, n \\
M_{i}=\mu_{i+1} / \mu_{i}, & i=1,2, \ldots, n-1 \\
\quad \varepsilon=H_{0} / L & i=1,2, \ldots, n \\
q_{i}, & \\
f^{*}(x) & \\
H_{1} . &
\end{array}
$$


The resulting expression for the residual $R_{i j}^{(R)}$ is:

$$
\begin{aligned}
R(j)= & \int_{0}^{1} \frac{h_{i}^{m+1}-h_{i}^{m}}{\Delta t} \phi_{j} \frac{\mathrm{d} x}{\mathrm{~d} \xi} \mathrm{d} \xi+\left[A_{i} h_{i}+\frac{B_{i} h_{i}^{2}}{2}\right. \\
& \left.+\frac{h_{i}^{3}}{6} \frac{\partial p_{i}}{\partial x}\right]\left.^{(m+1)}\right|_{x=0} ^{x=1 \text { or } x_{r}}-\int_{0}^{1}\left[A_{i} h_{i}+\frac{B_{i} h_{i}^{2}}{2}\right. \\
& \left.+\frac{h_{i}^{3}}{6} \frac{\partial p_{i}}{\partial x}\right]^{(m+1)} \frac{\mathrm{d} \phi_{i}}{\mathrm{~d} x} \frac{\mathrm{d} x}{\mathrm{~d} \xi} \mathrm{d} \xi, \quad i=1,2, \ldots, n,
\end{aligned}
$$

where $\Delta t$ is the time-step and the superscripts $(m+1)$ and $m$ denote $(m+1)$ th and $m$ th time-steps respectively. Equation (4), integrated across the thickness at the inlet, yields:

$$
\begin{aligned}
\int_{0}^{h_{i}} v_{x_{i}} \mathrm{~d} z_{i}=q_{i}=A_{i} h_{i}+\frac{B_{i} h_{i}^{2}}{2}+\left.\frac{h_{i}^{3}}{6} \frac{\partial p_{i}}{\partial x}\right|_{x=0}, \\
i=1,2, \ldots, n,
\end{aligned}
$$

which replaces the boundary term in eq. (21). The essential boundary condition of zero exit pressure for coextrusion and the natural boundary condition of zero pressure gradient for coating [see eq. (13)] are imposed at the outlet at $x=1$ or $x_{f}$.

The residual of eq. (11), after applying the divergence theorem, can be expressed as:

$$
\begin{aligned}
R_{i j}^{(N)}= & \int_{0}^{1}\left(p_{i}-M_{i} p_{i+1}\right)^{m+1} \phi_{j} \frac{\mathrm{d} x}{\mathrm{~d} \xi} \mathrm{d} \xi \\
& +\left.\left[\frac{\varepsilon^{3}}{C a_{i}} \frac{\partial h_{i}}{\partial x}\right]^{(m+1)}\right|_{x=1 \text { or } x_{i}} ^{x=0} \\
- & -\frac{\varepsilon^{3}}{C a_{i}} \int_{0}^{1} \frac{\partial h_{i}^{m+1}}{\partial x} \frac{\mathrm{d} \phi_{j}}{\mathrm{~d} x} \frac{\mathrm{d} x}{\mathrm{~d} \xi} \mathrm{d} \xi, \\
& i=1,2, \ldots, n-1 .
\end{aligned}
$$

In the case of coextrusion, the boundary term is replaced by eq. (16) at $x=0$. For coating, the additional boundary condition of eq. (17) is imposed.

The residuals of the remaining equation (12) are:

$$
R_{j}^{(T)}=\int_{0}^{1}\left(\sum_{i=1}^{n} h_{i}^{m+1}-f^{*}(x)\right) \phi_{j} \frac{\mathrm{d} x}{\mathrm{~d} \xi} \mathrm{d} \xi,
$$

for coextrusion, and

$$
\begin{aligned}
R_{n j}^{(N)}= & \int_{0}^{1} p_{n}^{m+1} \phi_{j} \frac{\mathrm{d} x}{\mathrm{~d} \xi} \mathrm{d} \xi+\left.\left[\frac{\varepsilon^{3}}{C a_{n}} \frac{\partial h_{n}}{\partial x}\right]^{(m+1)}\right|_{x=0} ^{x=x_{r}} \\
& -\frac{\varepsilon^{3}}{C a_{n}} \int_{0}^{1} \frac{\partial h_{n}^{m+1}}{\partial x} \frac{\mathrm{d} \phi_{j}}{\mathrm{~d} x} \frac{\mathrm{d} x}{\mathrm{~d} \xi} \mathrm{d} \xi,
\end{aligned}
$$

for coating.

The nonlinear system of algebraic equations (21), (23), and (24) or (25) can be expressed as:

$$
\mathbf{R}\left(\mathbf{u}^{(m+1)}\right)=\mathbf{0},
$$

where

$$
\mathbf{u}=\left[p_{11}^{(m+1)}, \ldots, p_{n 1}^{(m+1)}, h_{11}^{(m+1)}, \ldots, h_{n 1}^{(m+1)}, \ldots\right]
$$

is the vector of the nodal unknowns and $R$ is the column vector of the weighted residuals. This system of nonlinear equations is solved by Newton iteration at each time-step such that

$$
\mathbf{J}\left(\mathbf{u}_{k}^{(m+1)}\right)\left(\mathbf{u}_{k+1}^{m+1)}-\mathbf{u}_{k}^{(m+1)}\right)=-\mathbf{R}\left(\mathbf{u}_{k}^{(m+1)}\right),
$$

where $\mathbf{J}=\partial \mathbf{R} / \partial \mathbf{u}$ is the Jacobian matrix and the subscript $k$ represents the value evaluated at the $k$ th Newton iteration. The set of linear equations in eq. (27) is solved by the frontal routine developed by Hood (1976) for asymmetric matrices. For each timestep, the solution of the previous time-step is used as the initial guess in Newton iteration.

Until now, we have not considered the evaluation of the unknowns $A_{i}$ and $B_{i}$ involved in the Reynolds lubrication equations. For these unknowns, the additional set of equations are provided by eq. (5) or (6). If this additional set of equations was to be solved simultaneously with eqs (8) and (11) for the unknowns $h_{i}, p_{i}, A_{i}$, and $B_{i}$, the total degrees of freedom per element would be $12 n-$ an excessively high number of unknowns for a one-dimensional problem. (For example, if the flow of five layers was to be studied, then the number of unknowns in each element would be 60.) Therefore, to reduce computational time and cost, it was essential to reduce the number of unknowns as much as possible. In order to achieve this goal, the tridiagonal structure of eq. (5) or (6) is used to our advantage. This set of equations is decoupled from the rest and solved by the standard, rapid computational technique described in Carnahan et al. (1969). Thus, during Newton iteration, for given $h_{i_{k}}$ and $p_{i_{k}}$ in each element from the previous iteration, the unknowns $A_{i}$ and $B_{i}$, as well as their derivatives $\partial A_{i} / \partial u_{j k}$ and $\partial B_{i} / \partial u_{j k}$ with respect to the unknowns $u_{j k}$, are first computed as described in Appendix B. The quadratic convergence of Newton iteration is conserved in the numerical scheme.

This optimization scheme reduces the number of unknowns per element from $12 n$ to $6 n$. A typical computation with 20 elements of five-layer flow requires approximately $50 \mathrm{CPU}$ seconds on an Apollo DN-4000 work station for each time-step. In the absence of this scheme, the computational time and the cost were found to increase by at least two-fold. The optimization is expected to become more and more efficient as the total number of unknowns increases due to either an expanded flow domain or the large number of layers.

In the time-dependent analysis, the thickness profile is perturbed sinusoidally such that:

$$
\begin{aligned}
h_{i}(x, t=0)=h_{i_{e}}(x)[1+ & \left.h_{\mathrm{ampl}} \sin (2 \pi N x)\right], \\
& i=1,2, \ldots, n-1 \text { or } n,
\end{aligned}
$$

where $N$ is the wavenumber of the disturbance and $h_{i_{*}}(x)$ the thickness profile in the steady-state solution. With this initial perturbation, the transient analysis is carried out to compute the evolution of film thickness with time. Since the source of the disturbance can be any physical phenomenon such as the vibration of the die or the fluctuation in the flow rate of any layer, it can be well represented by the sinusoidal disturbance 
as in eq. (28). To keep the total thickness in coextrusion equal to the gap between the die walls, this perturbation is applied only to the thicknesses of $(n-1)$ layers, and the thickness of the $n$th layer is calculated using eq. (12). The amplitude of the disturbance, $h_{\mathrm{ampl}}$, is a parameter set to between $2 \%$ and $20 \%$ of the steady-state value. It is unnecessary to perturb the pressure $p_{i}$, because for a perturbed thickness a perturbed pressure will be automatically evaluated.

\section{RESULTS AND DISCUSSION}

\subsection{Multilayer extrusion}

To check the accuracy of the finite-element analysis, several tests were performed. At high $\mathrm{Ca}_{i}$, with a viscosity ratios of 1.0 for all the layers, the dimensionless pressure drop across the length of the die is 12.0, exactly matching the value obtained by the analytical expression for Hagen-Poiseuille flow. The pressure drop per unit length was also constant.

A constant pressure gradient along the die with uniform thickness was also observed at high $\mathrm{Ca}_{i}$ for up to seven-layer flow with several combinations of viscosity ratios. The ratios of the dimensionless pressures in adjacent layers were found to be equal to the inverses of the viscosity ratios. Then, according to the dimensional analysis using eq. (1), the same dimensional pressure exists in all layers at a given location. These numerical results are experimentally supported by Yu and Han (1973) for polymer melts using thin dies in the case of three-layer flow.

Analytical expressions for the flow rates and the thicknesses were derived for power-law liquids in twolayer and symmetric three-layer flow by Han (1981). For a power-law exponent equal to 1.0 (Newtonian liquids), the flow rates and the thicknesses obtained by the analytical expressions agree to within $0.01 \%$ with the numerical results.

Two typical thickness profiles for five-layer flow are shown in Fig. 4. The parameters are $M_{i}=0.2,0.2,5.0$, 5.0; $C a_{i}=10.0,2.0,0.4,2.0 ; q_{i}=0.1,0.05,0.5,0.05,0.3$; and $\varepsilon=0.1$. The gap between the walls is constant in Fig. 4(a) and varies linearly in Fig. 4(b). The viscosity ratios $M_{i}$ are selected such that layers 1 and 5 , and 2 and 4 have the same viscosities. This flow configuration is common in extrusion of food-packaging film in which a small amount of glue is extruded in layers 2 and 4 , and recycled scrap at high flow rate is extruded in one of the outer layers. It is assumed that the surface tension is the same for all the liquids. Then, $C a_{i}=C a_{i-1} M_{i-1}$ for $i=1,2, \ldots, n-1$. The proportions of the thicknesses occupied by various layers clearly remain constant over the domain of flow. Similar thickness profiles are obtained for up to seven layers and over a wide range of flow rates that follow the constraint in eq- (15). The viscosity ratios were changed over the range of 0.001 to 20.0 , and $\mathrm{Ca}_{i}$ over the range of $10^{-6}$ to 1000 . Since we impose the flow rate of each layer as a natural boundary condition in our analysis, the thicknesses are directly correlated to the flow rates of individual layers. (a)

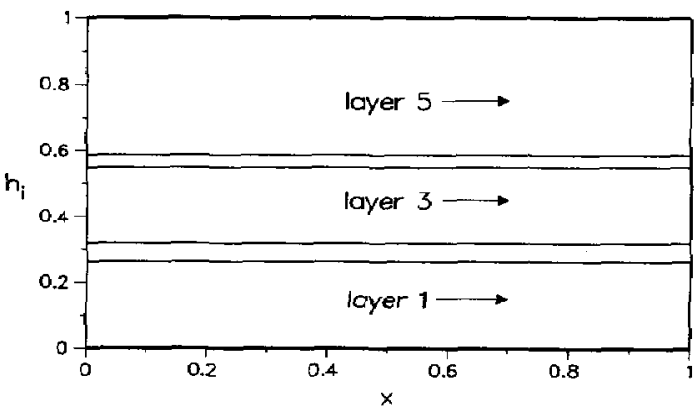

(b)

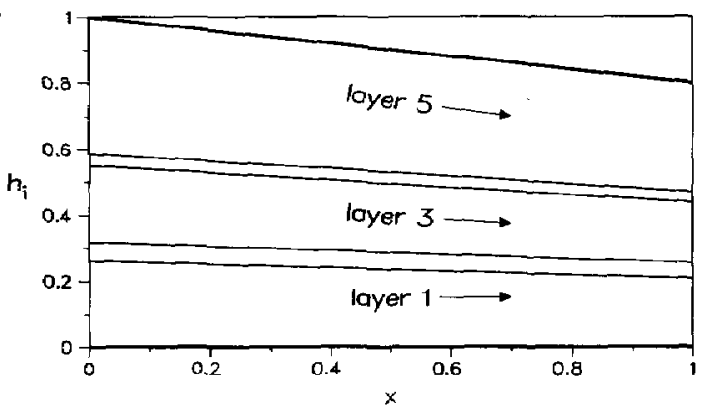

Fig. 4. Thickness profile of five-layer flow. The parameter's are $q_{t}=0.1,0.05,0.5,0.05,0.3 ; C a_{i}=10.0,2.0,0.4,2.0$; $M_{1}=0.2,0.2,5.0,5.0 ; \varepsilon=0.1$. (a) The gap between the walls is constant. (b) The gap between the walls is linearly varying along the length.

The effect of the viscosity ratios on the thicknesses and the volumetric fractions is shown in Fig. 5 . Three layers are used with the configuration, which is symmetric along the direction of the thickness $\left(q_{1} / q_{3}=1.0\right)$. The outer layers have the same viscosity. The gap between the die walls is constant. The other parameters are $C a_{1}=10.0, C a_{2}=M_{1} C a_{1}$, and $\varepsilon=0.1$. The change in $\left(h_{1}+h_{3}\right)$ with respect to $\left(q_{1}+q_{3}\right)$ is much higher at low volume fractions for low $M_{1}$. However, at high volume fractions, as the viscosity ratio increases, the slope in Fig. 5 increases.

In Fig. 6, the thickness ratios $h_{1} / h_{2}$ and $h_{1} / h_{3}$ are plotted against the volumetric ratio $q_{1} / q_{2}$ for various

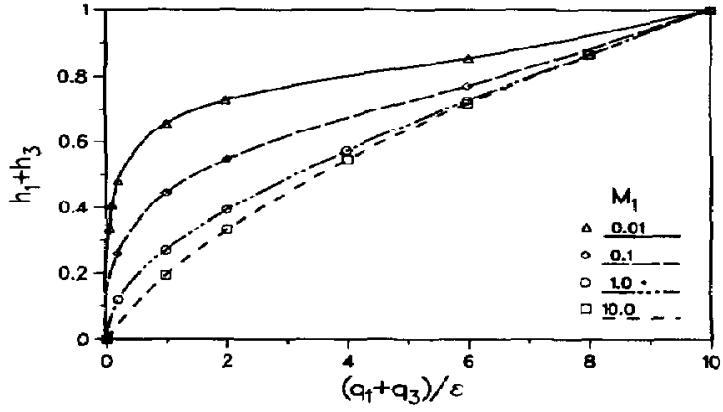

Fig. 5. The thickness $\left(h_{1}+h_{3}\right)$ vs. the volumetric fraction $\varepsilon\left(q_{1}+q_{3}\right)$ for three layers of symmetric configuration at various viscosity ratios $M_{1}$. The other parameters are $C a_{1}=10.0 ; C a_{2}=M_{1} C a_{1} ; \varepsilon=0.1 ; M_{2}=1 / M_{1}$. The gap between the die walls is constant. 
(a)

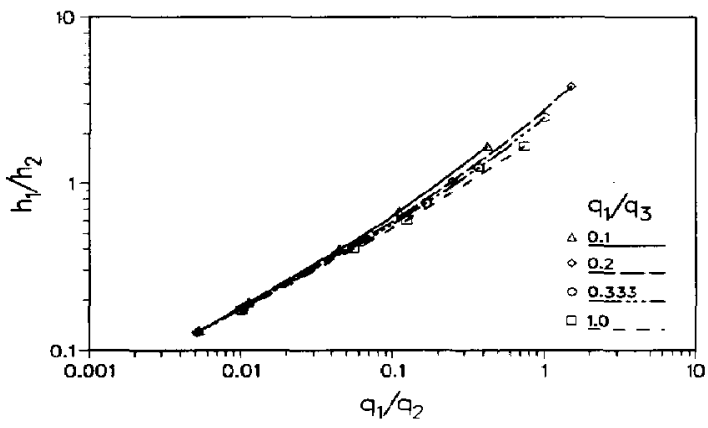

(b)

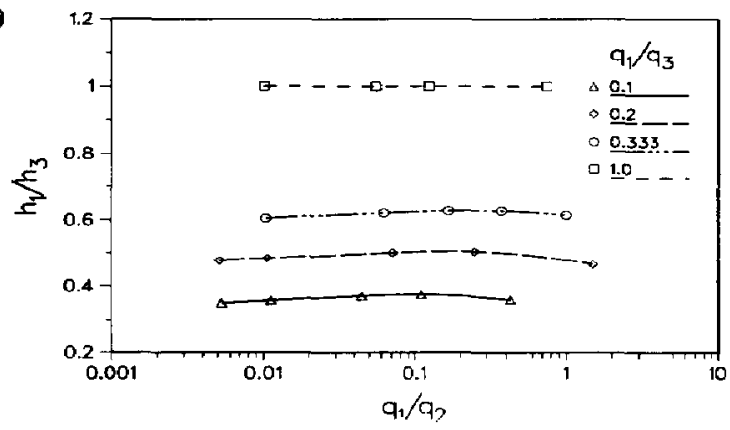

Fig. 6. The thickness ratios vs. the ratio of the volumetric flow rate $q_{1} / q_{2}$, for three-layer flow at various values of $q_{1} / q_{3}$. The other parameters are $M_{i}=0.1,10.0 ; C a_{1}=10.0$; $C a_{2}=M_{1} C a_{1} ; \varepsilon=0.1$. The gap between the die walls is constant.

values of $q_{1} / q_{3}$ for three-layer flow. Since $q_{1} \neq q_{3}$, the outer layers are asymmetric-usually the case when the recycled scrap is introduced into one of the outer layers. The other parameters are $M_{i}=0.1,10.0$; $C a_{i}=10.0,1.0$; and $\varepsilon=0.1$. The gap between the die walls is constant. The values of $h_{1}$ and $h_{3}$ interchange when the value of $q_{1} / q_{3}$ is inverted. Therefore, the values of $q_{1} / q_{3}>1$ are not considered in the analysis. For a given $q_{1} / q_{2}, h_{1} / h_{2}$ clearly remains approximately constant over a wide range of $q_{1} / q_{3}$. This observation also holds true for the various sets of viscosity ratios. Similarly, $h_{1} / h_{3}$ remains approximately constant over a range of $q_{1} / q_{2}$ for a given $q_{1} / q_{3}$, provided that the outer layers have the same viscosities.

Results similar to those in Figs 5 and 6 are important in establishing correlations between the thicknesses and the volumetric flow rates. In general, the thicknesses of the layers in the final products are dictated by the end-uses. However, in industrial operations, the flow rate of each layer can be controlled but not the thickness. Therefore, the correlation between the thicknesses and the flow rates must be established in $n$-layer coextrusion. We achieve this goal by choosing the boundary condition on the flow rate for each layer and then computing the thickness.

As discussed in section 3 , a sinusoidal disturbance of a specified amplitude is applied to the steady-state thickness profile as an initial condition and the timedependent analysis is carried out by backward- differencing. A time-step is chosen such that any further reduction in the time-step does not change the solution at any particular time. One such evolution of thickness profile with respect to time is shown in Fig. 7 for three-layer flow. The other parameters are $M_{i}=0.1, \quad 10.0 ; \quad q_{i}=0.1, \quad 0.5, \quad 0.4 ; \quad C a_{1}=10.0 ;$ $C a_{2}=M_{1} C a_{1}$; and $\varepsilon=0.1$. The amplitude of the initial disturbance is $5 \%$ of the steady-state solution. The gap between the die walls is kept constant. The initially disturbed thickness profile quickly approaches and remains at the steady-state value. The disturbance travels with constant amplitude along the length of the die, indicating that the system is neutrally stable. Similar observations were made for the range of viscosity ratios equal to $0.001-20.0$, for the range of $C a_{i}$ equal to $10^{-6}-100.0$, for the range of $q_{i}$ equal to $0.005-0.995$, and for the linearly varying gap between the die walls with slopes up to $0.4 \varepsilon$ for up to seven layers. The amplitude of the disturbance $h_{\text {ampl }}$ is varied from $2 \%$ to $20 \%$ of the steady-state solution, and the wavenumber $N$ is varied between 0.5 and 10.0. In all the investigated cases, the flow was neutrally stable.

Since in this analysis we have used the lubrication approximation, the inertia effects are neglected. The linear stability analyses of plane Poiseuille flow of two or three layers of different viscosities, carried out by Yih (1967) and Joseph et al. (1984), suggest that the flow is unstable in certain configurations at $R \boldsymbol{R} \rightarrow \mathbf{0}$. However, when inertia effects are neglected (i.e., in their analysis, when $R e=0$ ), the exponential growth factor in their linear stability analyses was zero. Therefore, according to the linear stability analysis, for negligible inertia effects, the flow is neutrally stable for Newtonian liquids. The above theoretical prediction is supported by our transient analysis. Moreover, the conclusion of neutrally stable flow is extended for multiple layers with various configurations over a wide range of viscosity ratios, capillary numbers and flow rates. Obviously, the known instabilities related to the inertia and to the density stratification are not observed in our analysis due to the limitations of the lubrication approximation.

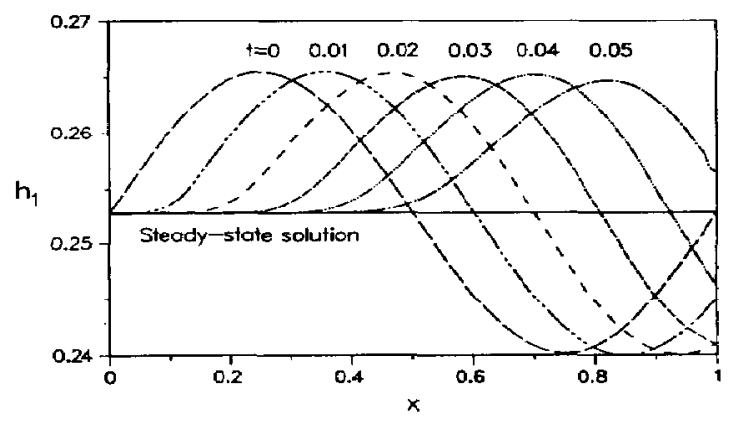

Fig. 7. Evolution of thickness profile with time for initial sinusoidal disturbance to three layers. The other parameters are $M_{1}=0.1 ; \quad M_{2}=1 / M_{1} ; C a_{1}=10.0 ; C a_{2}=M_{1} C a_{1}$; $\varepsilon=0.1 ; q_{i}=0.1,0.5,0.4 ; \Delta t=0.001 ; h_{\text {ampl }}=0.05$. The gap between the dic walls is constant. 


\subsection{Multilayer coating}

In this analysis, we examine only the downstream development of the coating film, and not the entire coating flow. In all the computations on multilayer coating, the total thickness at $x=0.0$ is fixed at 1.1 . The finite-element domain is defined from $x=0$ to $x=x_{f}$ such that the total thickness approaches a value of 1.0 at $x=x_{\mathrm{f}}$. In Fig. 8, the thickness profiles of two-layer coating are shown. The viscosity ratio $M_{2}$ in Fig. 8(a) is 0.01 and in Fig. 8(b) is 10.0. The other parameters are $C a_{i}=10.0,10^{-2} ; \varepsilon=0.1$; and $q_{i}=0.8,0.2$. The thickness profiles approach constant values asymptotically at $x=1.0$, which are equal to the volume fractions of each layer. Thus, as expected, the plug-flow region is approached at the downstream of multilayer coating. In the case of high viscosity ratio, this approach is slower than in the case of low viscosity ratio. However, the thickness profile in the plug-flow region is independent of the viscosity ratio.

The surface tension of the liquid in the outer layer also plays an important role in multilayer coating. The effect of the capillary number of the outer layer on the profile of the total thickness is studied in Fig. 9 for five-layer flow. In Fig. 9(a), the viscosity ratios and the capillary numbers are $M_{i}=0.2,0.2,5.0,5.0$ and $C a_{i}=10.0,2.0,0.4,2.0$; in Fig. $9(\mathrm{~b})$ they are $M_{i}=5.0$, 5.0, 0.2, 0.2 and $C a_{i}=1.0,5.0,25.0,5.0$. The other parameters are $q_{i}=0.1,0.05,0.5,0.05,0.3$; and $\varepsilon=0.1$. The length of the flow domain is extended up to $x_{\mathrm{f}}=2.0$ for the total thickness to reach a value of 1.0. As the surface tension of the outer layer increases, that is, as $\mathrm{Ca}$ decreases, the rate of approach to the plug-flow region decreases. For negligible surface ten-

(a)

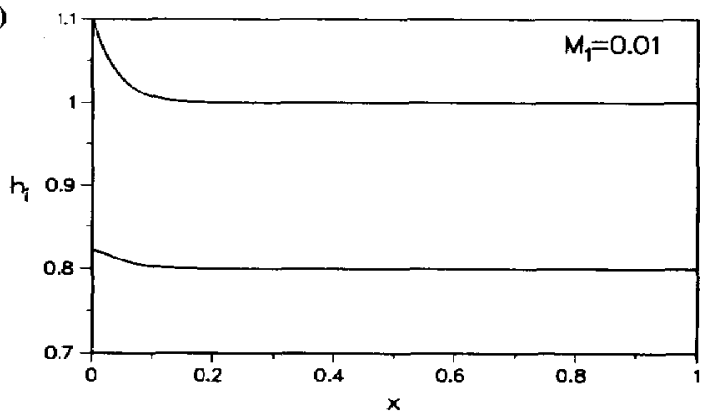

(b)

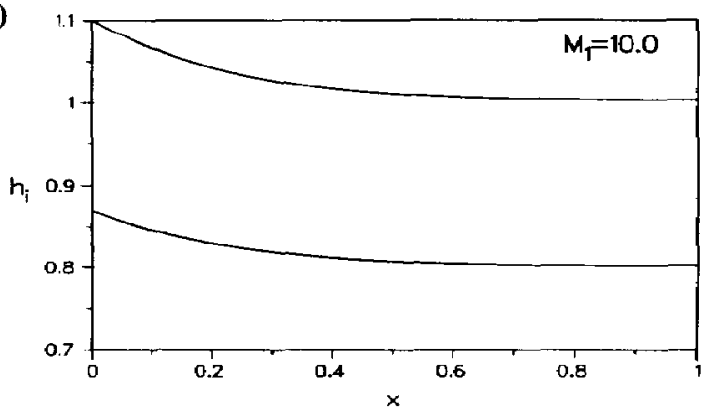

Fig. 8. Thickness profile of two-layer coating. The parameters are $C a_{i}=10.0,10^{-2} ; q_{i}=0.8,0.2$. (a)

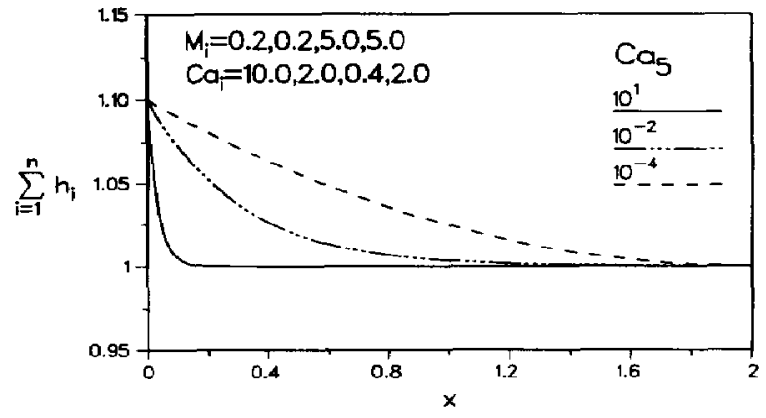

(b)

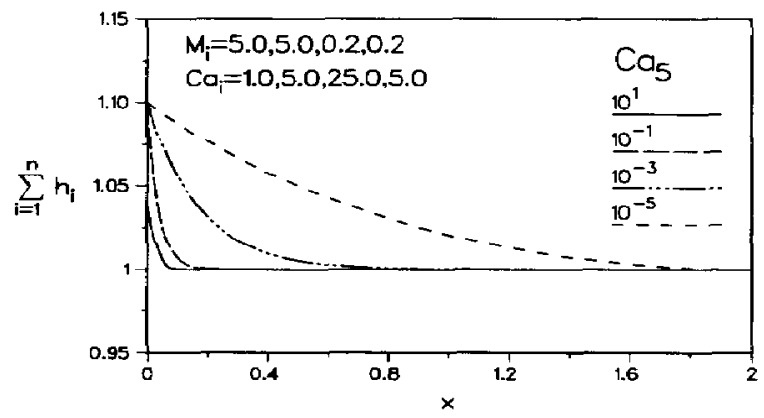

Fig. 9. The effect of the capillary number of the outer layer on the profile of the total thickness in five-layer coating. The parameters are $q_{i}=0.1,0.05,0.5,0.05,0.3 ; \varepsilon=0.1$.

sion of the inside layers, from eq. (11), it is expected that the curvature should increase with $\mathrm{Ca}_{5}$ for the same pressure. Similar to the conclusion in Fig. 8 , for the same $C a_{5}$, the approach to the plug-flow region is slower for the flow of low viscosity liquid in contact with air.

The effects of the surface tension between the liquids of the multilayer coating can also be studied. The thickness profile of the inner layer in two-layer flow is plotted in Fig. 10 for various values of $\mathrm{Ca}_{1}$. In Fig. 10(a), the viscosity ratio $M_{1}$ is 0.1 and in Fig. 10(b) it is 10.0. The other parameters are $q_{i}=0.8$, $0.2 ; \varepsilon=0.1$, and $C a_{2}=10^{-2}$. The thickness $h_{1}$ remains constant at $x=0$ for various values of $\mathrm{Ca}$. However, the approach to the plug-flow region is gradual for low values of $\mathrm{Ca}$ for constant $\mathrm{Ca}$. Similar effects of $\mathrm{Ca}_{1}$ on the total thickness profile are also observed. The comparison of Figs 9 and 10 suggests that the effect of the capillary numbers of the inner layers are not as pronounced as the capillary number of the outer layer. From this analysis, it is concluded that the capillary numbers, the viscosity ratios, and the flow rates of all the layers play important roles in determining the thickness profiles of all the layers. For polymer melts, the effects of any surface tension are negligible.

An initial sinusoidal disturbance is applied to the thickness profiles of all the layers and their evolution with time is investigated by solving the unsteady-state equations. Similar to coextrusion, a time-step is chosen such that any further reduction in time-step does not change the solution at a given time. In 
(a)

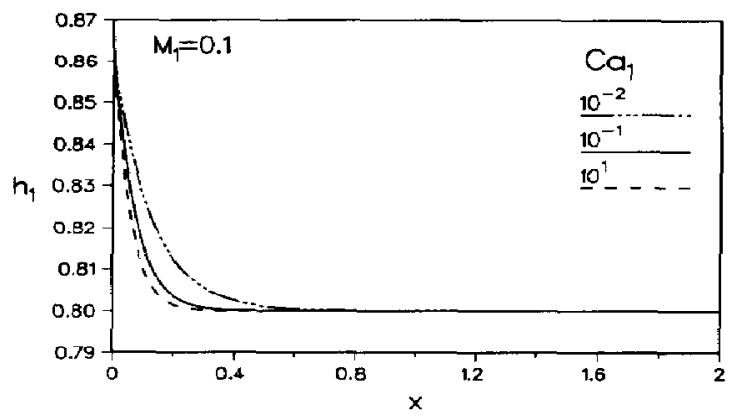

(b)

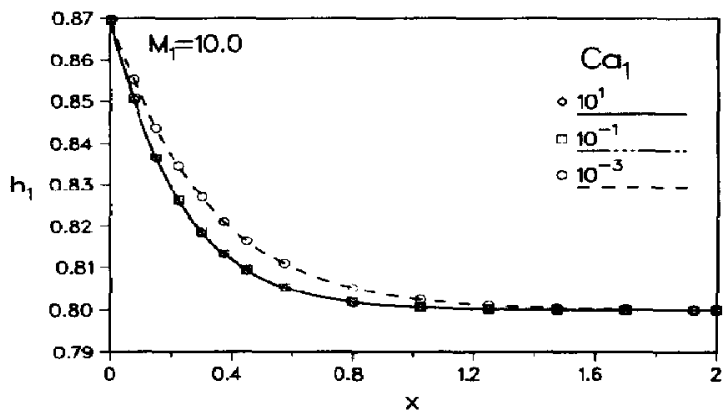

Fig. 10. The effect of the capillary number of the inner layer in two-layer flow. The parameters are $q_{i}=0.8,0.2 ; \varepsilon=0.1$; $C a_{2}=10^{-2}$.

Fig. 11, the total thickness profile is shown at various times for three-layer flow and the time-step is 0.001 . The other parameters are $M_{i}=0.1,10.0 ; q_{i}=0.2,0.4$, $0.4 ; C a_{i}=10.0,1.0,10^{-2}$; and $\varepsilon=0.1$. The amplitude of the initial disturbance equals $5 \%$ of the steady-state solution for the unknowns. The thickness profile approaches the steady-state value and remains there. The above computations are carried out over the same range of $M_{i}, C a_{i}, q_{i}, h_{\text {ampl }}$, and $N$ as those in the time-dependent analysis of multilayer extrusion. Similar to coextrusion, the stability of the multilayer coating flow can be attributed to the limitations of lubrication approximations and also to the fixed contact line.

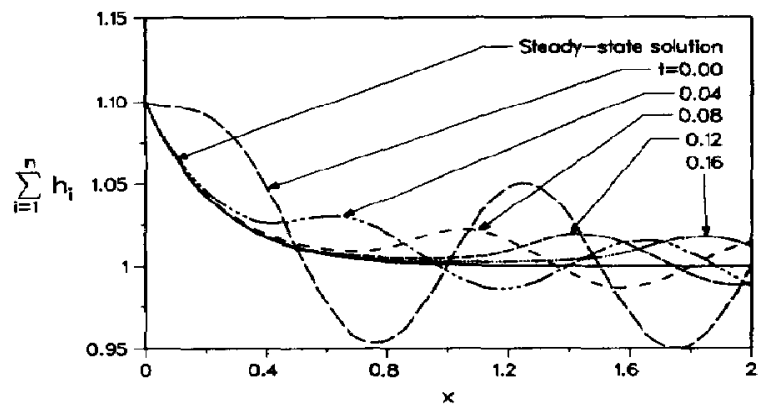

Fig. 11. Evolution of the total thickness profile with time for initial sinusoidal disturbance to three-layer flow. The parameters are $M_{i}=0.1,10.0 ; C a_{i}=10.0,1.0,10^{-2} ; \varepsilon=0.1$; $q_{t}=0.2,0.4,0.4 ; \Delta t=0.001 ; h_{\text {ampl }}=0.05$.
Besides, the ribbing instability observed in coating is a three-dimensional phenomenon that cannot be analyzed by two-dimensional disturbances considered here. In single-layer coating, Pearson (1960) used results from the lubrication analysis to carry out threedimensional linear stability analysis. When the wavenumber in his linear stability analysis was equal to zero, which is equivalent to two-dimensional disturbance, the growth factor was always negative (stable flow) or close to zero (neutrally stable flow) in a wedge-shaped spreader. Thus, the results of our nonlinear stability analysis of multilayer coating compare well with the linear stability analysis of singlelayer flow. A three-dimensional linear stability analysis is also feasible using the theory developed in this paper for multilayer flows.

All the above results of multilayer extrusion and of multilayer coating are based on the lubrication approximation. Therefore, inertia effects are neglected and the analysis is valid only for thin-film flows. Consequently, the interfacial instability observed in coextrusion due to nonzero inertia terms or due to density stratification cannot be predicted. Similarly, only the downstream development of the coating film could be analyzed by this analysis.

\section{CONCLUSIONS}

Thin-film flows of many layers in contact with each other are analyzed by means of the lubrication approximation. A convenient local coordinate transformation gives rise to algebraically simple equations and the Reynolds equations for $n$-layer flow evolve naturally. This unified system of equations is used to analyze multilayer extrusion and multilayer coating flows. In solving the equations, the computational cost is considerably reduced by .appropriately decoupling some of the equations.

In the steady-state analysis, the thickness and the pressure profiles were computed for various operating conditions and material properties such as the viscosities and the surface tensions. In the case of coating. the surface tension of the outer layer has the most pronounced effect on the thickness profile, compared to the effects of the surface tension of the inner layers. Surface tension is not expected to affect the thickness profiles under typical coextrusion conditions.

The flow was found to be stable over a wide range of investigated operating conditions and rheological parameters within the limitations of the lubrication approximation, which does not account for destabilizing inertia effects.

Acknowledgement-This research is being supported by a grant from the Dow Chemical Company, Midland, Michigan. Their support is gratefully acknowledged.

\section{NOTATION}

$a, b, c, d$ constant coefficients in eq. (B1)

$A, B \quad$ integration constants in eq. (4)

$\mathrm{Ca}$ capillary number

$f \quad$ dimensionless total thickness 


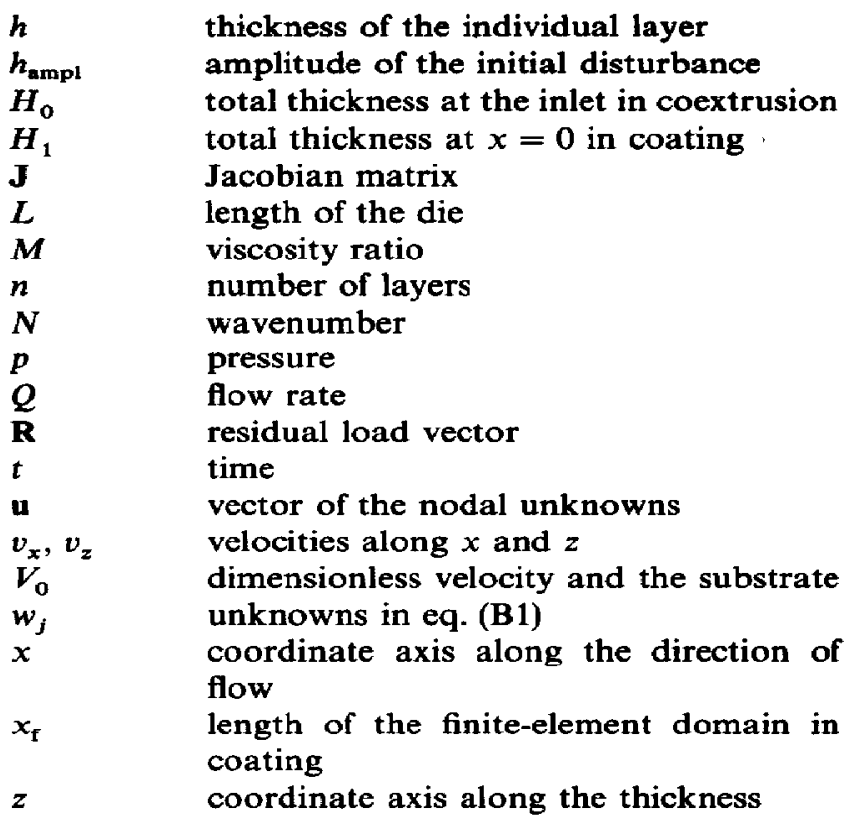

Greek symbols

$\beta, \gamma \quad$ variables in Appendix $B$

$\varepsilon \quad$ aspect ratio

$\mu \quad$ viscosity

$\xi \quad$ coordinate axis in isoparametric domain

$\rho$ density

$\sigma \quad$ surface tension

$\phi \quad$ basis function

\section{Superscripts}

* dimensionless quantities

$m \quad m$ th time-step

\section{Subscripts \\ $i \quad i$ th layer \\ $j \quad j$ th node}

\section{REFERENCES}

Batchelor, G. K., 1967, An Introduction to Fluid Dynamics. Cambridge University Press, Cambridge.

Bixler, N. E., 1982, Stability of a coating flow. Ph.D. Thesis, The University of Minnesota, Minneapolis.

Carnahan, B., Luther, H. A. and Wilkes, J. O., 1969, Applied Numerical Methods. John Wiley \& Sons, New York.

Coyle. D. J., Macosko, C. W. and Scriven, L. E., 1986, Film-splitting flows in forward roll coating. J. Fluid Mech. 171, 183-207.

Han, C. D., 1981, Multiphase Flow in Polymer Processing. Academic Press, New York.

Han, C. D. and Kim, Y. W. 1976, Further observations of the interface shape of conjugate fibers. J. appl. Polym. Sci. 20, 2609-2614.

Hood, P., 1976, Frontal solution program for unsymmetric matrices. Int. J. Numer. Meth. Engng 10, 379-393.

Joseph, D. D., Renardy, M. and Renardy, Y. 1984, Instability of the flow of two immiscible liquids with different viscosities in a pipe. J. Fluid Mech. 141, 309-317.

Kistler, S. F. and Scriven, L. E. 1984, Coating flow theory by finite-element and asymptotic analysis of the Navier-Stokes system. Int. J. Numer. Meth. Fluids 4, $207-229$.
Langlois, W. E., 1964, Slow Viscous Flows. Macmillan, New York.

Pearson, J. R. A., 1960, The instability of slow viscous flow under rollers and spreaders. J. Fluid Mech. 7, 481-500.

Reynolds, $O$., 1886, Papers on mechanical and physical aspects. Phil. Trans. $R$. Soc. 177, 157.

Schrenk, W. J., 1974, Multilayer film from a single die. Plastics Engng 30, 65-68.

Schrenk, W. J. and Alfrey, T., Jr., 1973, Coextruding multilayer blown film-Part I. SPE J. 29, 39-42.

Schrenk, W. J. and Alfrey, T., Jr., 1978, Coextruded multilayer polymer films and sheets, in Polymer Blends (Edited by Paul, D. R. and Seymour, N.), Vol. 2, pp. 129-165. Academic Press, New York.

Southern, J. H. and Ballman, R. L. 1973, Stratified bicomponent flow of polymer melts in a tube. J. appl. Polym. Sci. 20, $175-183$.

Strang, G. and Fix, G. J., 1973, An Analysis of the Finite Element Method. Prentice-Hall, Englewood Cliffs, NJ.

Tipei, N., 1962, Theory of Lubrication. Stanford University Press, Stanford, CA.

Waters, N. D., 1983, The stability of two stratified power-law liquids in Couette flow. J. Non-Newt. Fluid Mech. 12 , $85-94$.

White, J. L., Ufford, R. C., Dharod, K. R. and Price, R. L., 1972, Experimental and theoretical study of the extrusion of two-phase molten polymer system. J. appl. Polym. Sci. 16, 1313-1330.

Wong, W. T. and Jeng, C. C., 1987, The stability of two concentric non-Newtonian fluids in circular pipe flow. $J$. Non-Newt. Fluid Mech. 22, 359-380.

Yih, C. S., 1967, Instability due to viscosity stratification. $J$. Fluid Mech. 27, 337-352.

Yu, T. C. and Han, C. D., 1973, Stratified two-phase flow of molten polymers. J. appl. Polym. Sci. 17, 1203-1225.

\section{APPENDIX A}

In dimensionless form,

$$
\begin{gathered}
\mathbf{n}=\frac{-\varepsilon \frac{\partial h_{i}}{\partial x} \mathbf{i}+\mathbf{k}}{\left(1+\left(\varepsilon \frac{\partial h_{i}}{\partial x}\right)^{2}\right)^{1 / 2}} \approx-\varepsilon \frac{\partial h_{i}}{\partial x} \mathbf{i}+\mathbf{k}, \\
\mathbf{t}=\frac{\varepsilon \frac{\partial h_{i}}{\partial x} \mathbf{k}+\mathbf{i}}{\left(1+\left(\varepsilon \frac{\partial h_{i}}{\partial x}\right)^{2}\right)^{1 / 2}} \approx \varepsilon \frac{\partial h_{i}}{\partial x} \mathbf{k}+\mathbf{i},
\end{gathered}
$$

where $n$ and $t$ are the unit normal and tangent vectors to the surface and $i$ and $k$ are the unit vectors along the $x$ - and $z$-directions respectively. The shear stress is defined as $(n \cdot T) \cdot t$, where $\mathbf{T}$ is the total stress tensor. Thus,

$$
\begin{aligned}
(\mathbf{n} \cdot \mathbf{T}) \cdot \mathbf{t}= & -\varepsilon \frac{\partial h}{\partial x} T_{x x}+T_{x z}-\varepsilon^{2}\left(\frac{\partial h}{\partial x}\right)^{2} T_{x z}+\varepsilon \frac{\partial h}{\partial x} T_{z z} \\
= & -\varepsilon \frac{\partial h}{\partial x}\left[2 \frac{\partial v_{x}}{\partial x}-\frac{p}{\varepsilon^{2}}\right]+\left(\frac{1}{\varepsilon} \frac{\partial v_{x}}{\partial z}-\varepsilon \frac{\partial v_{z}}{\partial x}\right) \\
& +\varepsilon \frac{\partial h}{\partial x}\left(2 \frac{\partial v_{z}}{\partial z}-\frac{p}{\varepsilon^{2}}\right) .
\end{aligned}
$$

The pressure terms cancel out and the largest terms of $O(1 / \varepsilon)$ are collected. Since the dimensionless $\partial h / \partial x$ is of $O(1)$, the shear stress can be expressed as

$$
(\mathbf{n} \cdot \mathbf{T}) \cdot \mathbf{t} \approx \frac{1}{\varepsilon} \frac{\partial v_{x}}{\partial z}
$$

The stresses are continuous across the interfaces, and therefore,

$$
\partial v_{x_{i}} / \partial z_{i}=M_{i} \partial v_{x_{i+1}} / \partial z_{i+1},
$$


whereas on the free surface, the shear stress is zero, i.e.,

$$
\partial v_{x_{n}} / \partial z_{n}=0
$$

\section{APPENDIX B}

Equation (5) or (6) can be represented in the form of a tridiagonal system:

$$
\begin{aligned}
b_{1} w_{1}+c_{1} w_{2} & =d_{1}, \\
a_{2} w_{1}+b_{2} w_{2}+c_{2} w_{3} & =d_{2}, \\
a_{3} w_{2}+b_{3} w_{3}+c_{3} w_{4} & =d_{3}, \\
\ldots & \\
a_{2 n} w_{2 n-1}+b_{2 n} w_{2 n} & =d_{2 n} .
\end{aligned}
$$

The unknowns $w_{j}$ in the above system of equations are correlated to the unknowns $A_{i}$ and $B_{i}$ in eq. (5) or (6) by the relations

$$
A_{i}=w_{j}, \quad B_{i}=w_{j+1},
$$

where $i=(j+1) / 2$ and $i=1,2, \ldots, n$. By comparing eq. (5) or (6) and eq. (B1):

$$
b_{j}, b_{j+1}, d_{j}, d_{j+1}=f\left(h_{i}, p_{i}\right)
$$

However, the coefficients $a_{j}$ and $c_{j}(j=1,2, \ldots, 2 n)$ are all constants.

The complete algorithm for the solution of $w_{j}$ in eq. (B1) is given by the series of recursive formulae, which, along with the formulae for the simultaneous computation of $\partial w_{j} / \partial u_{l}$, where $\mathrm{u}=\left[p_{1}, \ldots, p_{n}, h_{1}, \ldots, h_{n}\right]$, are given below.

Let us define $\beta_{j}$ and $\gamma_{j}(j=1,2, \ldots, 2 n)$ and their derivatives w.r.t. $u_{t}$ such that:

$$
\begin{aligned}
& \beta_{1}=b_{1}, \quad \gamma_{1}=\frac{d_{1}}{\beta_{1}}, \\
& \beta_{j}=b_{j}-\frac{a, c_{j-1}}{\beta_{j-1}}, \\
& \gamma_{j}=\frac{d_{j}-a_{j} \gamma_{j-1}}{\beta_{j}} \\
& l=1,2, \ldots, j+1 \text { (for odd } j \text { ), } \\
& \frac{\partial \beta_{j}}{\partial u_{i}}=\frac{\partial b_{j}}{\partial u_{l}}-\frac{a_{j} c_{j-1}}{\beta_{j-1}^{2}} \frac{\partial \beta_{j-1}}{\partial u_{l}} \\
& \frac{\partial \gamma_{j}}{\partial u_{l}}=\frac{\partial}{\partial u_{l}}\left[\frac{d_{j}-a_{j} \gamma_{j-1}}{\beta_{j}}\right], \\
& j=1,2, \ldots, 2 n \text {. }
\end{aligned}
$$

Using $\beta_{j}$ and $\gamma_{j}(j=1,2, \ldots, 2 n)$, the solution of $w_{j}$ in eq. (B1) is given by:

$$
\left.\begin{array}{c}
w_{2 n}=\gamma_{2 n}, \\
w_{j}=\gamma_{j}-\frac{c_{j} \gamma_{j+1}}{\beta_{j}}, \\
\frac{\partial w_{j}}{\partial u_{l}}=\frac{\partial \gamma_{j}}{\partial u_{I}}-c_{j} \frac{\partial}{\partial u_{l}}\left(\frac{\gamma_{j+1}}{\beta_{j}}\right),
\end{array}\right\} \begin{array}{r}
l=1,2, \ldots, 2 n, \\
j=(2 n-1),(2 n-2), .
\end{array}
$$

